\title{
Mouse Abdominal Fat Depots Reduced by Butyric Acid-Producing Leuconostoc mesenteroides
}

\author{
John Jackson Yang ${ }^{1,+}{ }^{+}$Minh Tan Pham ${ }^{2,+}{ }^{+}$Adelia Riezka Rahim ${ }^{2}$, Tsung-Hsien Chuang ${ }^{3}$, \\ Ming-Fa Hsieh ${ }^{4} \mathbb{D}$ and Chun-Ming Huang ${ }^{2, *}$ \\ 1 Department of Life Sciences, National Central University, Taoyuan 32001, Taiwan; \\ johnjacksonyang@gmail.com \\ 2 Department of Biomedical Sciences and Engineering, National Central University, Taoyuan 32001, Taiwan; \\ minhtan19906@gmail.com (M.T.P.); adeliariska07@gmail.com (A.R.R.) \\ 3 Immunology Research Center, National Health Research Institutes, Zhunan, Miaoli County 35053, Taiwan; \\ thchuang@nhri.edu.tw \\ 4 Department of Biomedical Engineering, Chung Yuan Christian University, Taoyuan 32023, Taiwan; \\ mfhsieh@cycu.edu.tw \\ * Correspondence: chunming@ncu.edu.tw; Tel.: +886-3-422-7151 (ext. 36101) \\ + These authors contributed equally to this work.
}

Received: 16 June 2020; Accepted: 31 July 2020; Published: 3 August 2020

check for updates

\begin{abstract}
The activation of peroxisome proliferator-activated rece ptor gamma (PPAR- $\gamma$ ) is known to induce the differentiation of adipocytes. This study aimed to investigate the probiotic effect of Leuconostoc mesenteroides (L. mesenteroides) on high-fat diet (HFD)-induced PPAR- $\gamma$ activation and abdominal fat depots. Incubation of differentiated 3T3-L1 adipocytes with media of L. mesenteroides EH-1, a butyric acid-producing strain, significantly reduced the amounts of lipid droplets. The oral administration of L. mesenteroides EH-1 produced large amounts $(>1 \mathrm{mM})$ of butyric acid in cecum and attenuated the HFD-induced upregulation of PPAR- $\gamma$ and accumulation of abdominal fats in mice. The combination of $2 \%$ glucose with L. mesenteroides $\mathrm{EH}-1$ increased the production of butyric acid and potentiated the probiotic activity of L. mesenteroides EH-1 against the formation of lipid droplets in 3T3-L1 adipocytes as well as abdominal fats in HFD-fed mice. The inhibition of free fatty acid receptor 2 (Ffar2) by its antagonist, GLPG-0974, markedly diminished the probiotic effects of L. mesenteroides EH-1 plus glucose on the suppression of HFD-induced PPAR- $\gamma$ and abdominal fats. Besides demonstrating the probiotic value of L. mesenteroides EH-1, our results highlight the possible therapy targeting the butyric acid-activated Ffar2 pathway to reduce abdominal fats.
\end{abstract}

Keywords: abdominal fats; butyric acid; high-fat diet; Leuconostoc mesenteroides; PPAR- $\gamma$

\section{Introduction}

The accumulation of fat in abdominal adipose depot is related to the development of obesity-related cardiometabolic risk, referring to the chances of having type 2 diabetes, heart disease or stroke [1]. Together, overweight and obesity are the fifth leading cause of death in the world, causing nearly 3.4 million deaths annually [2]. Genomic insights into the multiple factors that control the abdominal fat deposition have been proposed [3]. Adipocytes require several transcription factors, such as peroxisome proliferator-activated receptor gamma (PPAR- $\gamma$ ), CCAAT/enhancer-binding proteins (C/EBPs), signal transducers and activators of transcription (STATs) and Krüppel-like factor (KLF) proteins to facilitate the differentiation of adipocyte precursor cells into mature adipocytes [4]. PPAR- $\gamma$ is a transcriptional factor belonging to the ligand-activated nuclear receptor superfamily, which directly regulates the expression of genes involved in adipocyte differentiation, lipid and carbohydrate metabolism and 
adipokine synthesis. Adipokine released by adipose tissues is a key factor linking obesity-induced inflammation. PPAR- $\gamma$ activation induces the preadipocyte differentiation to adipocytes and stimulates triglyceride storage [5].

A high-fat diet (HFD) is largely used in animal research to induce obesity and study-related comorbidities. Although obesity results from many pathologic factors, such as genetics, unhealthy lifestyl, and eating habits, it has been proved that changes in the compositional pattern of the gut microbiota contribute in central ways to the development of various metabolic disorders. The gut microbiota regulates many host physiologic processes, including utilization of glucose, early dissociation and dihydroxylation of bile acid and biosynthesis of vitamins and amino acids [6]. Conversely, obesity further worsens the dysbiosis of gut microbiota, forming a vicious circle. Thus, the rectification of gut dysbiosis has become a promising strategy to treat obesity by drug or nutritional intervention [7]. Short-chain fatty acids (SCFAs), the main fermentation metabolites of probiotic bacteria such as butyric acid, have been shown to reduce HFD-induced metabolic disorders and inflammation [8]. Different fibres as prebiotics give rise to different amounts and patterns of the main SCFAs: Acetic, propionic and butyric acids. SCFAs are natural ligands for free fatty acid receptor 2 and 3 (Ffar2/3), found on a wide range of cell types, including adipocytes $[9,10]$. The various types of SCFAs with different affinities to Ffar2/3 contribute to diverse physiological effects semicolon. Ffar2, also known as G (Gi) protein-coupled receptor 43 (GPR43), is expressed in human adipose tissue and mediates the lipolytic properties of SCFAs [11]. The potency rank order of SCFAs for Ffar2 is acetic acid propionic acid $>$ butyric acid $>$ valeric acid $>$ formic acid [10]. Butyric acid is a vital energy source for the colonic epithelial cells and has an inhibitory effect on the growth of cancer cells in vitro [12]. Butyric acid is also thought to mediate the lipid metabolism by slowing down fat transport from the intestine [13].

Leuconostoc mesenteroides (L. mesenteroides), a heterofermentative lactic acid bacterium, is particularly well adapted to sugary niches and therefore possesses a wide spectrum of biocatalytic activities for carbohydrate modifications. L. mesenteroides and its enzymes have been used to produce carbohydrates and derivatives such as dextran and fructose [14]. Leuconostoc species are epiphytic bacteria that are commonly found in the natural environments and have been used as a probiotic bacterium for food fermentations [15]. For instance, L. mesenteroides starter cultures are used in some dairy and bread dough. We isolated a butyric acid-producing L. mesenteroides EH-1 strain from Mongolian curd cheese. Our previous study demonstrated that L. mesenteroides EH-1 can reduce blood glucose and increase the insulin in the model of type 1 diabetic mice induced by streptozotocin [16]. In this study, we fed mice with HFD to augment the abdominal fat depots and investigated whether Ffar2 mediates the regulation of L. mesenteroidesEH-1 on accumulation of visceral fats. The Ffar2-mediated pathway activated by butyric acid-producing probiotic bacteria may reveal novel molecules for targeted drug therapy against visceral fat depots.

\section{Materials and Methods}

\subsection{Ethics Statement}

Institute of Cancer Research (ICR) female mice (8-9 weeks old) were purchased from the National Laboratory Animal Center, Taipei, Taiwan. Experiments were carried out in strict accordance with an approved Institutional Animal Care and Use Committee (IACUC) protocol at National Central University (NCU), Taiwan with an approved protocol (NCU-106-016, 19 December 2017). Mice were sacrificed via inhalation of $\mathrm{CO}_{2}$ anaesthesia.

\subsection{Glucose Fermentation of L. mesenteroides EH-1}

For fermentation, L. mesenteroides EH-1 bacteria $\left(10^{7}\right.$ colony-forming unit (CFU)/mL) were incubated in rich media with the composition of $0.001 \%(w / v)$ of phenol red (Sigma, St. Louis, MO, USA), $1.5 \mathrm{~g} / \mathrm{L}$ of $\mathrm{KH}_{2} \mathrm{PO}_{4}, 10 \mathrm{~g} / \mathrm{L}$ of $\mathrm{K}_{2} \mathrm{HPO}_{4}, 5 \mathrm{~g} / \mathrm{L}$ ofTryptic Soy Broth (TSB) (Sigma, St. Louis, MO, USA) and $10 \mathrm{~g} / \mathrm{L}$ of yeast extract (Biokar Diagnostics, Beauvais, France) with or without $2 \%(20 \mathrm{~g} / \mathrm{L}) \mathrm{of}$ 
glucose. Bacteria were incubated for $24 \mathrm{~h}$ at $37^{\circ} \mathrm{C}$. Rich media in the absence of L. mesenteroides $\mathrm{EH}-1$ bacteria with or without $2 \%$ glucose were included as controls. Phenol red was used as an indicator to monitor bacterial fermentation. The transformation of colour change in rich media, from red to yellow, indicating fermentation, was quantified by measuring the optical density at $562 \mathrm{~nm}\left(\mathrm{OD}_{562}\right)$. The reduction of $\mathrm{OD}_{562}$ was detected when fermentation occurred.

\subsection{T3-L1 Cell Differentiation and Oil Red O Staining}

The 3T3-L1 preadipocytes (ATCC CL-173) within 3-7 passages were cultured in Dulbecco's Modified Eagle's medium (DMEM) (Gibco-BRL, Grand Island, NY, USA) with 10\% (v/v) foetal bovine serum (FBS) (Irvine Scientific, Santa Ana, CA, USA), $10 \mathrm{mmol} / \mathrm{L}$ of L-glutamine, $1 \mathrm{mmol} / \mathrm{L}$ of sodium pyruvate, $100 \mathrm{unit} / \mathrm{mL}$ of penicillin and $100 \mu \mathrm{g} / \mathrm{mL}$ of streptomycin. The differentiation of 3T3-L1 preadipocytes $\left(0.2 \times 10^{5}\right.$ cells/well $)$ was induced by treatment of post-confluent cells with differentiation medium A (DMA) consisting of DMEM, $0.5 \mathrm{mM}$ of 1-methyl-3-isobutylxanthine (IBMX), $1.0 \mu \mathrm{M}$ of dexamethasone (DEX), $1 \mu \mathrm{M}$ of insulin (Sigma), 1\% penicillin-streptomycin and 10\% FBS. Each medium was then refreshed every 2 days for 10 days. Culture media $(100 \mu \mathrm{L} / \mathrm{mL})$ of the L. mesenteroides EH-1 bacteria in TSB with or without $2 \%$ glucose was added onto the culture of 3T3-L1 preadipocytes at $37^{\circ} \mathrm{C}$ for $30 \mathrm{~min}$ on Day $0,2,4,6,8$, and 10 . A Whatman nylon membrane with a $0.22-\mu \mathrm{m}$ pore size (GE Healthcare, Chicago, IL, USA) was used to filter culture media. Three wells for each treatment were conducted. The levels of lipids in 3T3-L1 preadipocytes were stained by an Oil Red O staining kit (Sigma). In brief, $4 \%$ formaldehyde fixed cells were stained with a working solution for $30 \mathrm{~min}$. Lipids stained red were imaged by light microscopy and extracted in $250 \mu \mathrm{L}$ of isopropanol for quantification via measuring absorbance at 510 using a Synergy HTX plate reader (BioTek Instruments, Winooski, VT, USA) [17].

\subsection{Feeding Mice with L. mesenteroides EH-1 and Ffar2 Inhibition}

L. mesenteroides EH-1 bacteria were cultured in rich medium for $24 \mathrm{~h}$ in $37^{\circ} \mathrm{C}$ and centrifuged at $6000 \times \mathrm{g} \mathrm{rpm}$ for $5 \mathrm{~min}$. The pellet was then diluted in phosphate-buffered saline (PBS). Mice were fed with $60 \%$ calorie HFD (60\% fat by weight., BioLASCO Taiwan Co., Ltd.) and administered $200 \mu \mathrm{L}$ of $2 \%$ glucose, L. mesenteroides EH- 1 ( $10^{7}$ CFU) or L. mesenteroides EH-1 plus glucose by oral gavage at an interval of 3 days for 25 days. For inhibition of Ffar2, GLPG-0974 (Tocris Bioscience, Bristol, UK), a Ffar2 antagonist, was dissolved in $0.1 \%$ dimethyl sulfoxide (DMSO) to make a stock solution. GLPG-0974 (1 mg/kg body weight) was diluted in saline then was given at $1 \mathrm{~mL} / \mathrm{kg}$ body weight [18] every 3 days for 25 days by oral gavage right before administration of L. mesenteroides EH-1 plus $2 \%$ glucose. Body weights were measured every three days. Mice were fasted for $4-8 \mathrm{~h}$ on the last day of treatment. The level of blood glucose from the tail blood was measured using a glucometer [16]. The abdominal fat mass was photographed and weighted. Five mice per group were used in each experiment.

\subsection{Butyric Acid Detection by High-Performance Liquid Chromatography (HPLC)}

Mouse cecum was collected after feeding mice with HFD and oral gavage of L. mesenteroides EH-1 in the presence or absence of $2 \%$ glucose every day for four days. Cecum was centrifuged at $5000 \times g$ $\mathrm{rpm}$ for $10 \mathrm{~min}$. The supernatants were filtered through a $0.22-\mu \mathrm{m}$ microfiltration membrane to remove bacteria and all insoluble particles. The filtrates were vortexed and equilibrated at room temperature for $5 \mathrm{~min}$. Thereafter, $100 \mu \mathrm{L}$ of concentrated $\mathrm{HCl}$ was added, followed by a vortex mixing step of $15 \mathrm{~s}$. The samples were extracted for $20 \mathrm{~min}$ using $5 \mathrm{~mL}$ of diethyl ether. After centrifugation, the supernatant was transferred to a Pyrex extraction tube before $500 \mu \mathrm{L}$ of a $1 \mathrm{~mol} / \mathrm{L}$ solution of $\mathrm{NaOH}$ was added. The aqueous phase was transferred to an autosampler vial and $100 \mu \mathrm{L}$ of concentrated $\mathrm{HCl}$ were added. The analysis of butyric acid was performed using an Agilent 1200 series HPLC system with a ZORBAX Eclipse XDB-C18 column $(4.6 \times 250 \mathrm{~mm}, 5 \mu \mathrm{m})$ [16]. The mobile phase consisted of $20 \mathrm{mmol} / \mathrm{L} \mathrm{NaH}{ }_{2} \mathrm{PO}_{4}$ solution ( $\mathrm{pH}$ 2.2) and acetonitrile. The detector was set at $210 \mathrm{~nm}$. 
The concentrations of butyric acid were calculated according to calibration curves of a butyric acid analytical standard.

\subsection{PPAR- $\gamma$ Expression}

The 3T3-L1 cells were grown in DMEM or differentiation media for six days. Differentiated 3T3-L1 cells were treated with or without $0.1 \mu \mathrm{M}$ of GLPG-0974 in the presence of media collected from culture of $100 \mu \mathrm{L} / \mathrm{mL}$ L. mesenteroides EH-1 bacteria $\left(10^{7} \mathrm{CFU} / \mathrm{mL}\right)$ and $2 \%$ glucose for $30 \mathrm{~min}$ on Day $0,2,4$, and 6 . Cells were lysed with radioimmunoprecipitation assay (RIPA) buffer (Thermo Fisher Scientific). Mouse abdominal fat depots were ground and lysed with tissue lysis buffer containing protease inhibitors. Protein $(30 \mu \mathrm{g})$ was loaded to a $10 \%$ sodium dodecyl sulphate-polyacrylamide gel electrophoresis (SDS-PAGE) gel, then transferred into a polyvinylidene difluoride (PVDF) membrane (Sigma) and blocked with 5\% (w/v) nonfat milk before overnight incubation with primary antibody to PPAR- $\gamma$ (1:1000), or $\beta$-actin (1:5000). Thereafter, the blotting was incubated with horseradish peroxidase (HRP)-conjugated secondary antibody (goat anti-mouse (1:5000) Thermo Fisher Scientific) for $1 \mathrm{~h}$. Chemiluminescent detection reagent (Thermo Fisher Scientific) and Omega Lum C Imaging System (Gel Co., San Francisco, CA, USA) were used to detect protein bands, and then analyzed with ImageJ software $1.50 \mathrm{~b}$ (National Institutes of Health, Bethesda, MD, USA).

\subsection{Statistical Analysis}

Data analysis was performed by unpaired t-test. The $p$-values of $<0.05\left(^{*}\right),<0.01\left({ }^{* *}\right)$, and $<0.001$ $(* * *)$ were considered significant. The mean \pm standard deviation (SD) was calculated from at least three separate experiments.

\section{Results}

\subsection{Reduction of Lipid Droplets in Differentiated 3T3-L1 Adipocytes by L. mesenteroides EH-1}

Our previous studies revealed that L. mesenteroides EH-1 is a butyric acid-producing bacterial strain when cultured in the presence of glucose in vitro [16]. As shown in Figure 1a,b, yellowish media and a decrease in $\mathrm{OD}_{562}$ were detected in the culture of L. mesenteroides EH-1 $\left(10^{7} \mathrm{CFU} / \mathrm{mL}\right)$ for $12 \mathrm{~h}$ in rich media which contained $2.5 \%$ glucose in TSB $(0.161 \pm 0.006)$. Media exhibited a more yellowish colour and the $\mathrm{OD}_{562}$ value declined considerably upon the addition of $2 \%$ glucose into rich media of $12 \mathrm{~h}$ culture of L. mesenteroides EH-1 $(0.104 \pm 0.008)$. The data further confirmed our earlier results that glucose can trigger and enhance the fermentation of L. mesenteroides EH-1. We previously demonstrated that butyric acid was detectable in the culture media of L. mesenteroides EH-1 [16]. We next investigated whether the culture media can affect the formation of lipid droplets during the differentiation process of adipocytes. The number of lipid droplets stained by Oil Red O in 3T3-L1 preadipocytes was largely increased when DMEM was replaced with differentiation media in the cell culture, demonstrating the differentiation of 3T3-L1 cells induced by differentiation media. The incubation of differentiated 3T3-L1 cells with culture media of L. mesenteroides EH-1 for 30 min largely reduced the number of lipid droplets (Figure 1c,d). The addition of $2 \%$ glucose into the bacterial culture enhanced the ability of culture media to reduce the formation of lipid droplets. The result illustrated that metabolites in culture media of L. mesenteroides EH-1 influenced the differentiation of adipocytes. 
a

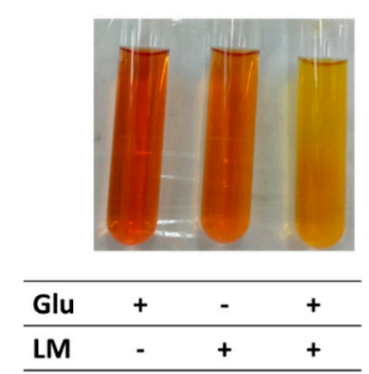

c

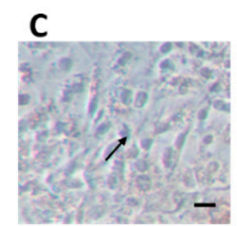

LM

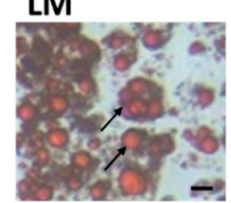

Glu

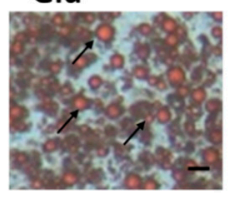

LM+Glu

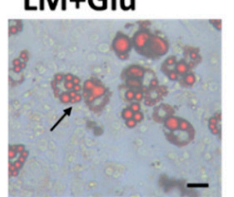

b

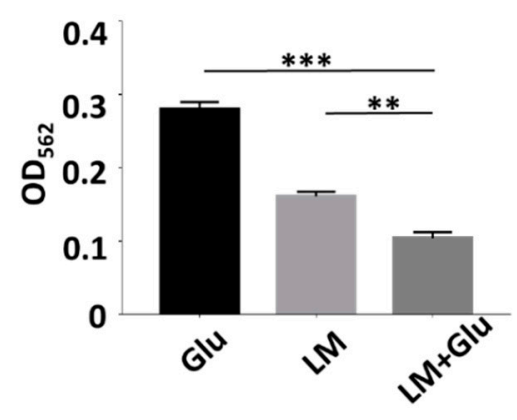

d

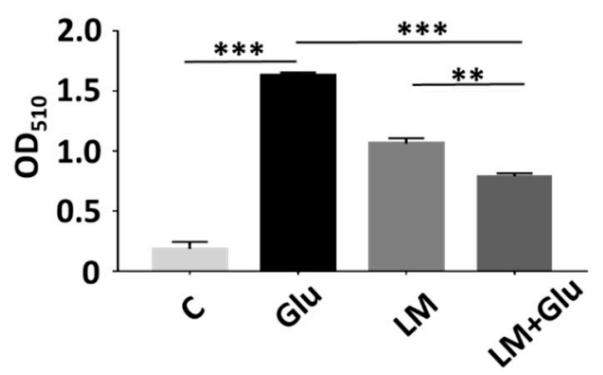

Figure 1. Inhibition of lipid accumulation of differentiated 3T3-L1 cells by L. mesenteroides EH-1. (a) L. mesenteroides (LM) was cultured in rich media with or without the addition of $2 \%$ glucose (Glu) for 12 h. Rich media added with $2 \%$ glucose alone were included as a control. Bacterial fermentation was indicated by the colour change of phenol red from red to yellow. (b) Media of culture with bacteria and/or glucose were measured by $\mathrm{OD}_{562}$. The reduction of $\mathrm{OD}_{562}$ was detected when fermentation happened. After $12 \mathrm{~h}$, the $\mathrm{OD}_{562}$ value of L. mesenteroides (LM) with glucose (Glu) was significantly reduced $(0.104 \pm 0.008, n=3)$ compared to that $(0.270 \pm 0.010, n=3)$ of rich media and glucose. (c) 3T3-L1 cells were grown in dimethyl sulfoxide (DMEM) (c) or differentiation media for 10 days. Differentiated 3T3-L1 cells were incubated with media collected from culture of L. mesenteroides EH-1 with or without $2 \%$ glucose for $30 \mathrm{~min}$. Lipid droplets (arrows) were stained by Oil Red O. (d) The contents of lipid droplets were quantified by measurement of $\mathrm{OD}_{510}$. The $\mathrm{OD}_{510}$ value $(0.793 \pm 0.021$, $n=3$ ) for detection of lipid droplets in differentiated 3T3-L1 cells incubated with L. mesenteroides (LM) with glucose (Glu) was significantly decreased compare to that $(1.629 \pm 0.027, n=3)$ of cells incubated with differentiation media with glucose. Data shown represent the mean \pm standard deviation (SD) of experiments performed in triplicate. ${ }^{* *} p<0.01$; ${ }^{* *} p<0.001$. Bar $=50 \mu \mathrm{m}$.

\subsection{In Vivo Production of Butyric Acid and Reduction of HFD-Induced Abdominal Fats by} L. mesenteroides EH-1

We next examined if $L$. mesenteroides EH-1 can produce butyric acid in vivo. HFD-fed ICR mice were given L. mesenteroides EH-1 via oral gavage every day for four days. Results from HPLC analysis (Figure 2a,b) showed that butyric acid concentrations of greater than $1 \mathrm{mM}$ were detected in the cecum of HFD-fed mice administered with L. mesenteroides EH-1 $(1.097 \pm 0.083 \mathrm{mM})$. Administration of glucose alone did not change the level of butyric acid in cecum $(0.181 \pm 0.095 \mathrm{mM})$. However, oral gavage of L. mesenteroides EH-1 along with $2 \%$ glucose significantly enhanced the butyric acid production of L. mesenteroides EH-1 in the cecum of HFD-fed mice $(1.591 \pm 0.102 \mathrm{mM})$. The result revealed the probiotic activity of $L$. mesenteroides EH-1, which may metabolize various carbon sources in cecum to produce the substantial butyric acid in vivo. Furthermore, supplementation of $2 \%$ glucose in the oral gavage of L. mesenteroides EH-1 can promote the in vivo production of butyric acid. In parallel, we found that abdominal fat depots in HFD-fed mice $(2.814 \pm 0.127 \mathrm{~g})$ were markedly reduced when mice were administered with L. mesenteroides EH-1 (1.838 $\pm 0.217 \mathrm{~g})$ for 25 days. The reduction of 
HFD-induced abdominal fats by L. mesenteroides EH-1 was augmented when $2 \%$ glucose was included in oral gavage of L. mesenteroides EH-1 (1.118 $\pm 0.111 \mathrm{~g})$ (Figure 2c,d). Moreover, supplementation of $2 \%$ glucose in the oral gavage of L. mesenteroides EH-1 reduced the body weight HFD-fed mice (Figure $\mathrm{S1}$ ). The result suggested butyric acid produced by L. mesenteroides EH-1 in cecum may regulate the HFD-induced formation of abdominal fat depots.
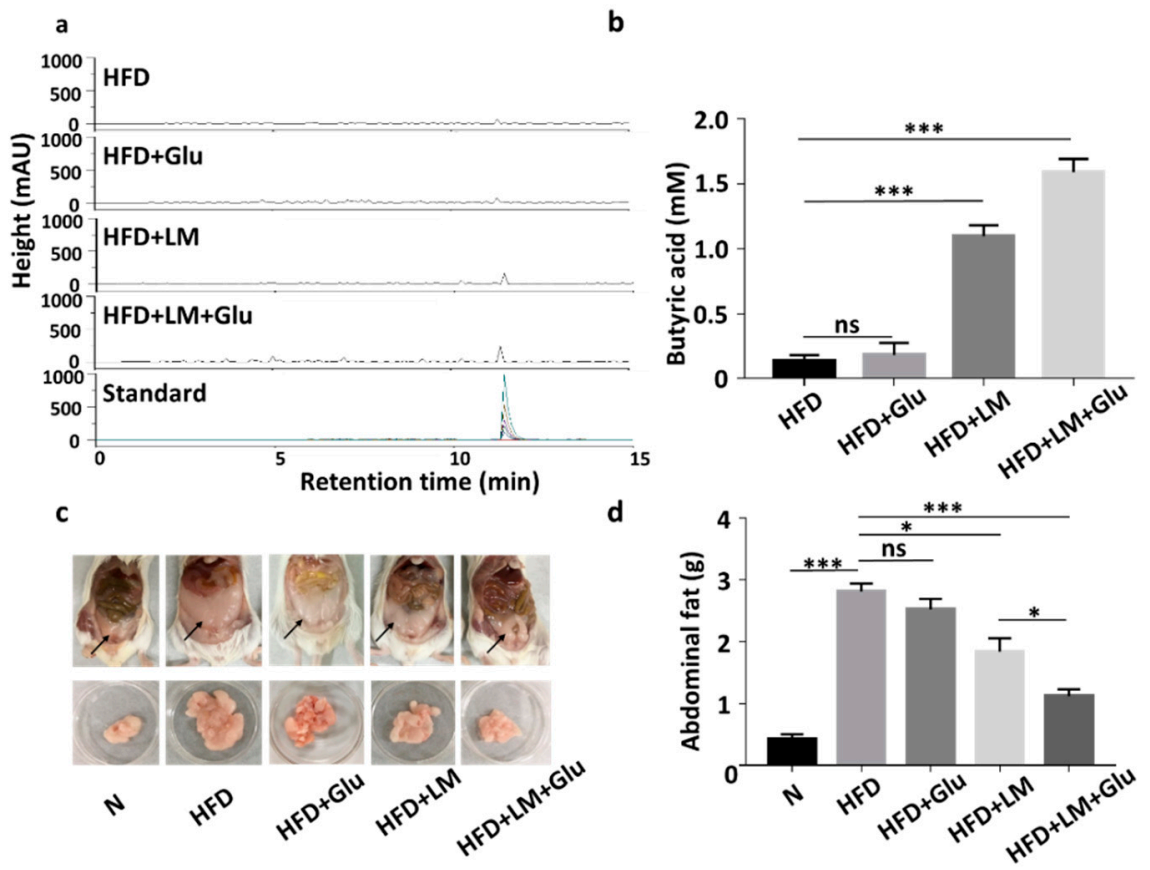

Figure 2. Butyric acid production of L. mesenteroides EH-1 in cecum and inhibition of abdominal fat depots in high-fat diet (HFD)-fed mice. (a) HFD-fed Institute of Cancer Research (ICR) mice were administered with L. mesenteroides EH-1 (LM) in the presence or absence of $2 \%$ glucose (Glu) via oral gavage every day for four days. The high-performance liquid chromatography (HPLC) chromatograms of butyric acid in mouse cecum were displayed. (b) The concentration (mM) of butyric acid was quantified based on the heights (milli-absorbance unit (mAU)) of standard peaks with concentrations of butyric acid from 0-20 mM. The level $(1.591 \pm 0.101, n=5)$ of butyric acid in mouse cecum after LM administration was significantly increased compared to that $(0.136 \pm 0.044, n=5)$ in HFD-fed mice. (c,d) Abdominal fat depots (arrows) in HFD-fed mice administered with L. mesenteroides EH-1 in the presence $(1.118 \pm 0.111, n=5)$ or absence $(1.838 \pm 0.217, n=5) 2 \%$ glucose for 25 days were dissected and weighted. Abdominal fat depots in mice fed with normal diet $(\mathrm{N})(0.426 \pm 0.072, n=5)$ were included for comparison. Data are represented as mean \pm SD of experiments performed in triplicate and five replicates. ${ }^{*} p<0.05 ;{ }^{* * *} p<0.001 ; \mathrm{ns}=$ nonsignificant. Bars $=10 \mathrm{~mm}$.

\subsection{Suppression of HFD-Induced PPAR- $\gamma$ Upregulation by L. mesenteroides EH-1}

In agreement with the literature that HFD increased the mRNA and protein levels of PPAR- $\gamma$ in mice [19], the protein level of PPAR- $\gamma$ in abdominal fats was increased by two-fold when ICR mice were fed with HFD for 25 days. The upregulation of PPAR- $\gamma$ by HFD was significantly suppressed in mice orally administered with L. mesenteroides EH-1, but not glucose alone (Figure 3). The addition of $2 \%$ glucose into the oral gavage of L. mesenteroides EH-1 enhanced the suppression of PPAR- $\gamma$. The result clearly illustrated the capability of butyric acid-producing L. mesenteroides EH-1 in abolishing the HFD-induced upregulation of PPAR- $\gamma$ in abdominal fats. 
a

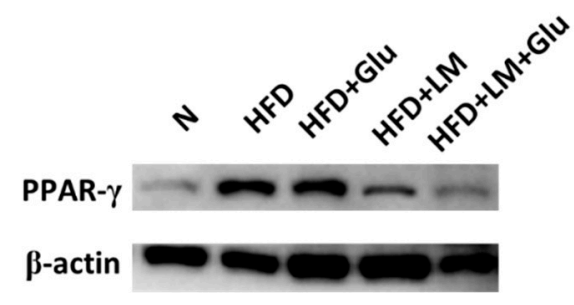

b

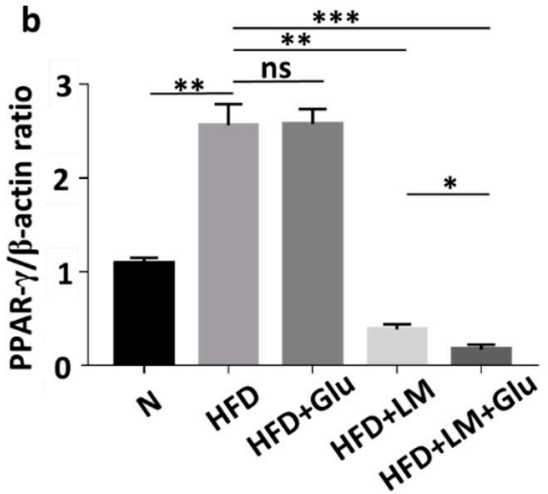

Figure 3. Attenuation of HFD-induced upregulation of PPAR- $\gamma$ by L. mesenteroides EH-1. (a) The levels of PPAR- $\gamma$ and $\beta$-actin in abdominal fat depots of mice fed with normal diet (N) or HFD along with glucose $(\mathrm{Glu})$, L. mesenteroides EH-1 (LM) or L. mesenteroides EH-1 plus glucose every 3 days for 25 days were detected by western blot analysis. (b) The ratio intensities of PPAR- $\gamma$ to $\beta$-actin were quantified. PPAR- $\gamma$ expression $(0.172 \pm 0.050, n=3)$ was reduced in HFD-fed mice with treatment of $L$. mesenteroides EH-1 and glucose (LM + Glu) compared to that $(2.565 \pm 0.224, n=3)$ in HDF-fed mice without treatment or that $(2.579 \pm 0.158, n=3)$ in HFD-fed mice with glucose. The mean \pm SD for three separate experiments was calculated. ${ }^{*} p<0.05 ;{ }^{* *} p<0.01 ;{ }^{* * *} p<0.001$. ns $=$ nonsignificant.

3.4. Effects of Ffar2 Inhibitor Administration with L. mesenteroides EH-1 Probiotic Diet on PPAR- $\gamma$ Level in High-Fat Diet Mice

As shown in Figure 2, oral administration of L. mesenteroides EH-1 with or without glucose can concurrently lead to the production of butyric acid in cecum and the reduction of abdominal fats in HFD-fed mice. It has been reported that butyric acid produced in the gut can enter the bloodstream and affect the physiological functions of various organs via activation of Ffar2 or Ffar3 [20]. We next examined whether Ffar2 mediates the action mechanism of butyric acid-producing L. mesenteroides EH-1 in suppression of HFD-induced upregulation of PPAR- $\gamma$ and reduction of abdominal fats. HFD-fed mice were orally administered with GLPG-0974 to inhibit Ffar2. As shown in Figure 4, administration of GLPG-0974 alone did not alter the protein expression of PPAR- $\gamma$ and the accumulation of abdominal fats in HFD-fed mice. Consistent with Figure 3, the protein expression of PPAR- $\gamma$ and abdominal fats was intensely reduced when HFD-fed mice were administered with L. mesenteroides EH-1 plus glucose every 3 days for 25 days. However, the reduction of PPAR- $\gamma$ expression and abdominal fats by L. mesenteroides EH-1 plus glucose were largely reversed $(2.957 \pm 0.076 \mathrm{~g})$ when GLPG-0974 was added into the oral gavage of L. mesenteroides EH-1 plus glucose (Figure 4). The result demonstrated that Ffar 2 was essential for L. mesenteroides EH-1 to attenuate the HFD-induced PPAR- $\gamma$ expression in abdominal fats and formation of fat depots. 
a
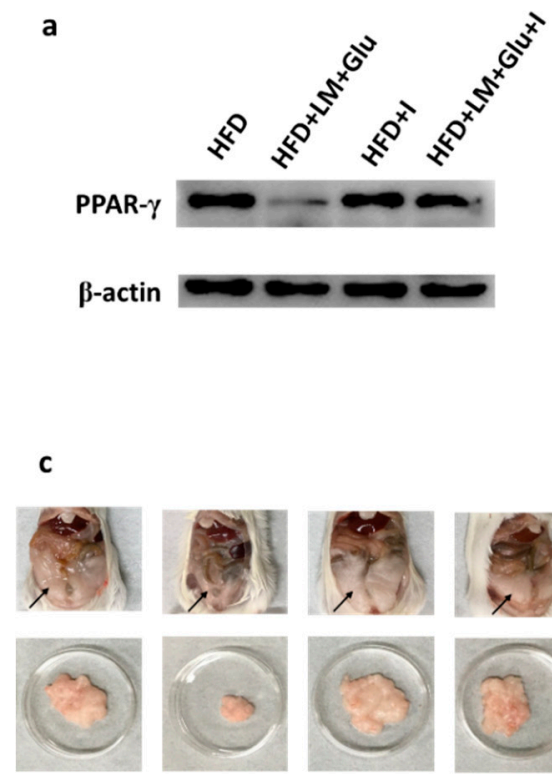

4रP
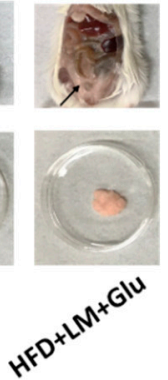

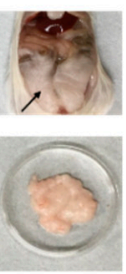

$4 \times 0^{x+1}$
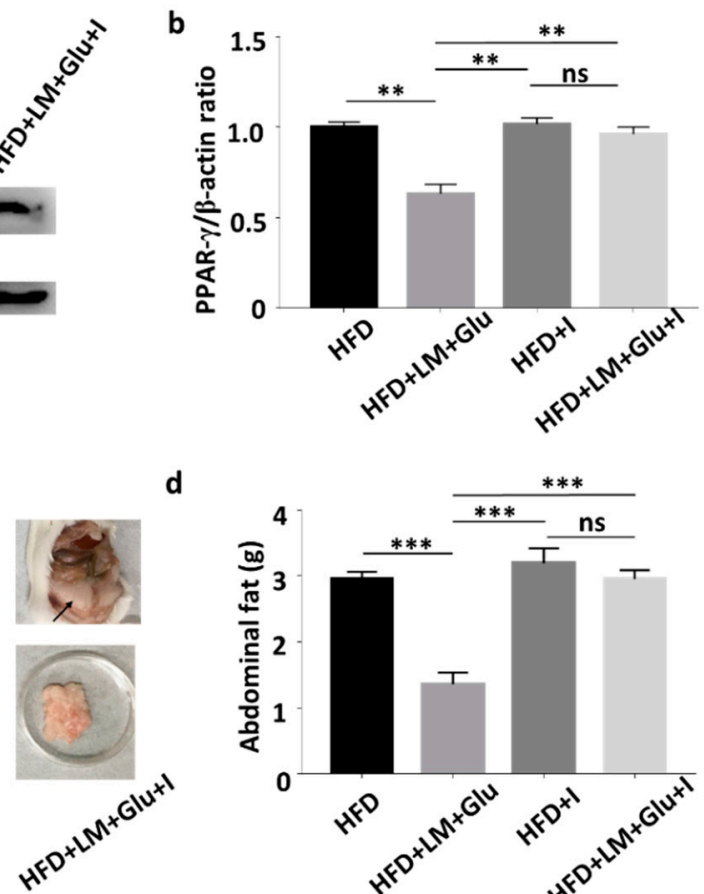

d

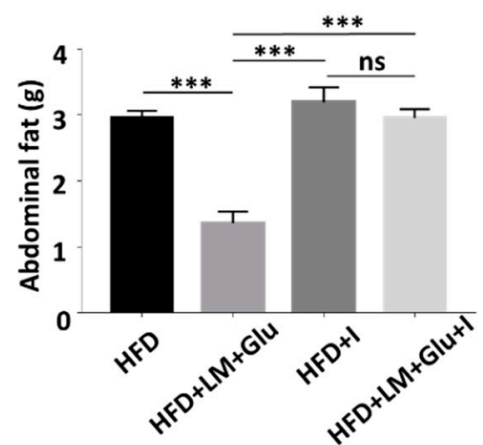

Figure 4. Involvement of Ffar2 in the action of L. mesenteroides EH-1 on the HFD-induced upregulation of PPAR- $\gamma$. HFD-fed mice were orally administered with or without L. mesenteroides EH-1 (LM) plus $2 \%$ glucose (Glu) right after administration of GLPG-0974, a Ffar2 antagonist (I), every 3 days for 25 days. (a) The levels of PPAR- $\gamma$ and $\beta$-actin in abdominal fat depots were detected by western blot analysis.

(b) The ratio intensities of PPAR- $\gamma$ to $\beta$-actin were quantified. (c) Representative mice with abdominal fats (arrows) in the whole body or isolated abdominal fat depots were shown. (d) Abdominal fat depots HFD-fed mice administered with $L$. mesenteroides EH- 1 with glucose in the presence $(2.957 \pm 0.076 \mathrm{~g}$, $n=5)$ or absence $(1.358 \pm 0.102 \mathrm{~g}, n=5)$ GLPG-0974 for 25 days were dissected and weighted. Data shown represent the mean \pm SD of experiments performed in triplicate and five replicates. ${ }^{* *} p<0.01$; *** $p<0.001$. ns $=$ nonsignificant. Bars $=10 \mathrm{~mm}$.

\section{Discussion}

Probiotic L. mesenteroides is currently used for food fermentation [21]. Here, we demonstrated, for the first time, that a butyric acid-producing L. mesenteroides EH-1 strain lowers the PPAR- $\gamma$ expression and abdominal fat accumulation in an HFD mouse model. Our results showed that the oral gavage of L. mesenteroides EH-1 alone without glucose produced high amounts of butyric acid and significantly inhibited HFD-induced abdominal fats (Figure 2). The L. mesenteroides EH-1 may metabolize endogenous carbon sources, such as starch in the gut [22], to undergo fermentation for the production of butyric acid. Luminal glucose concentrations in the small intestine in humans are approximately $50-500 \mathrm{mM}$ and exceed $300 \mathrm{mM}$ after a meal. For animals on nearly physiological diets, the luminal glucose concentrations averaged 0.4-24 $\mathrm{mM}$ [7]. Thus, luminal glucose may be an endogenous carbon source for L. mesenteroides EH-1. The carbohydrates in HFD may also serve as carbon sources for fermentation by L. mesenteroides EH-1 before the mouse gut. After the consumption of HFD, carbohydrates may be broken down to hexose monosaccharides such as glucose, fructose and galactose $[23,24]$ which become carbon sources for L. mesenteroides EH-1. It has been reported that faecal SCFAs detected in HFD-fed mice were mainly produced by gut microbiota [25].

The beneficial effects of SCFAs produced by fermentation of gut microbiota on treatments of adiposity, as well as reduction of lipid profiles, by promoting mitochondria function and energy consumption [26,27]. Body weight gain contributes to insulin-resistant glucose uptake [28]. SCFAs derived from fermentation of gut microbiota can modulate glucose homeostasis and increase insulin 
sensitivity [29]. However, the rapid decay of SCFAs in vivo within few hours in plasma showed the limitation of use of SCFAs as therapeutics [30]. A high concentration (greater than $1 \mathrm{mM}$ ) of butyric acid was detected in the cecum of mice fed with L. mesenteroides EH-1 with/without glucose (Figure $1 \mathrm{~b}$ ), demonstrating that L. mesenteroides EH-1 is a potent strain for producing butyric acid. Particularly, many reports have provided evidence that butyric acid reduced HFD-induced metabolic dysfunctions [31,32]. Studies recently reported that supplementation of butyric acid led to interrupt the PPAR- $\gamma$ signaling molecules [33]. Heimann et al. showed butyric acid decreased the rate of de novo lipogenesis in a dose-dependent manner in rat adipocytes [34]. Our results in Figure 4 supported that activation of Ffar2 by butyric acid produced by L. mesenteroides EH-1 was a key event that suppressed the HFD-induced PPAR- $\gamma$ expression and abdominal fats.

The expression of PPAR- $\gamma$ prior to other transcription factors in adipocytes modulates the adipogenesis in exponentially growing fibroblast cell lines, demonstrating the significance of PPAR- $\gamma$ in the regulation of adipocyte differentiation [35]. Consistent with other studies [35,36], our results demonstrated that differentiation media induced a significant increase in protein expression of PPAR- $\gamma$ in 3T3-L1 cells. Incubation of differentiated 3T3-L1 cells with medial collected from the culture of L. mesenteroides EH-1 with $2 \%$ glucose downregulated the differentiation induced an increase in PPAR- $\gamma$. The treatment of differentiated 3T3-L1 cells with GLPG-0974, a Ffar2 antagonist, restored the L. mesenteroides EH-1 and glucose-induced downregulation of PPAR- $\gamma$ (Figure S2). For in vivo experiments, HFD induced an upregulation of PPAR- $\gamma$ in abdominal fats in ICR mice (Figure 3). Overexpression of Ffar2 and PPAR- $\gamma$ target genes in adipose tissues was detected in HFD-fed mice [21]. This effect was associated with fat mass accumulation, increased adipocyte size and decreased basal lipolysis. It has been shown that HFD-fed Ffar2 knockout mice exhibited obesity correlated with high expressed mRNA level of PPAR- $\gamma$ in abdominal fat tissues [25]. Similarly, our study demonstrated that the protein level of HFD-induced PPAR- $\gamma$ in mice orally administered with L. mesenteroides EH-1 and GLPG-0974 was much higher than that in mice administered with L. mesenteroides EH-1 alone (Figure 4). Thus, the reduction of overexpressed Ffar2 may enhance the PPAR- $\gamma$-induced adipogenesis and elevate lipid accumulation [36]. In a previous study, Poirier et al. emphasized the treatment of obesity via the amelioration of PPAR- $\gamma$ expression in adipocytes [37]. Our current study provided evidence that a significant decrease in PPAR- $\gamma$ expression and abdominal fats in HFD-fed mice can be achieved by oral administration of probiotic L. mesenteroides EH-1 (Figures 3 and 4).

Visceral and subcutaneous fats are two different adipose tissues that are present in the abdominal cavity in humans [38]. Subjects with visceral abdominal obesity had lower glucose disposal and oxidation as well as greater lipid oxidation when compared to those with peripheral obesity [39]. It has been documented that 20 genes, which are mostly related to lipid metabolism and glucose homeostasis, are markedly different between two types of fats. Genes encoding angiotensinogen, resistin and adiponectin in visceral fat were expressed 5-fold, 3.8-fold and 12.2-fold, respectively, higher than those in subcutaneous fat. Moreover, PPAR- $\gamma$ in visceral fat is six-fold greater than that in subcutaneous fat. The visceral fat in abdominal region contains white adipose tissues, which are the fat stores associated with energetic and oxidative metabolism [40]. Moreover, Ffar2 is primarily expressed in white or abdominal adipocytes in mice compared to brown adipocytes [25]. Previous works showed that acetate and propionate inhibited isoproterenol-induced lipolysis in 3T3-L1 adipocytes [41], and the activation of Ffar2 by acetate in vivo resulted in reduced plasma levels of free fatty acids, showing the inhibition of lipolysis [42]. Our results showed that L. mesenteroides EH-1 can produce ample amounts of butyric acid in cecum and activate Ffar2 to mitigate the HFD-induced PPAR- $\gamma$ in abdominal fat (Figures 2-4). When combined with the results of previous studies, we envision that inhibition of HFD-induced PPAR- $\gamma$ via activation of Ffar2 by butyric acid produced by L. mesenteroides EH-1 may be an effective means to reduce the abdominal fat depot. 


\section{Conclusions}

The emerging contribution of intestinal microbes to the glucose homeostasis and lipid metabolism has made probiotic microbes possible for treatments of obesity [43,44]. The regulation of abdominal fat deposition via a probiotic uptake associated with lowering plasma glucose level is gaining scientific interest. In summary, we studied a probiotic strain, L. mesenteroides EH-1, which can produce high concentrations of butyric acid in vivo. We found that L. mesenteroides EH-1 activated Ffar2, downregulated PPAR- $\gamma$, and reduced abdominal fats in HFD-fed mice.

Supplementary Materials: The following are available online at http://www.mdpi.com/2076-2607/8/8/1180/s1, Figure S1: Effect of L. mesenteroides EH-1 on HFD-induced weight gain in mice. Figure S2: Involvement of Ffar2 in down-regulation of PPAR- $\gamma$ by L. mesenteroides EH-1 in the differentiated 3T3-L1 cells.

Author Contributions: J.J.Y., M.T.P. and C.M.H. wrote the manuscript. J.J.Y., M.T.P. and A.R.R. performed experiments. T.-H.C. and M.-F.H. assisted experimental designs. C.-M.H. designed experiments and edited the manuscript. All authors have read and agreed to the published version of the manuscript.

Funding: This study was supported by 106/107/108-Landseed Hospital-NCU joint grants and Ministry of Science and Technology (MOST) Grants (108-2622-B-008-001-CC1; 108-2314-B-008-003-MY3, and 107-2923-B-008-001-MY3).

Acknowledgments: The authors thank toHuiying Li in Ming-Fa Hsieh's laboratory at Chun Yuan Christian University, Taiwan for substantial help in HPLC analysis.

Conflicts of Interest: All co-authors do not have any financial conflicts of interest and the funder has not played any decision-making role in the research.

\section{References}

1. White, U.A.; Fitch, M.D.; Beyl, R.A.; Hellerstein, M.K.; Ravussin, E. Differences in in vivo cellular kinetics in abdominal and femoral subcutaneous adipose tissue in women. Diabetes 2016, 65, 1642-1647. [CrossRef]

2. Matsuzawa, Y.; Nakamura, T.; Shimomura, I.; Kotani, K. Visceral fat accumulation and cardiovascular disease. Obes. Res. 1995, 3, 645S-647S. [CrossRef] [PubMed]

3. Kobayashi, M.; Ohno, T.; Hada, N.; Fujiyoshi, M.; Kuga, M.; Nishimura, M.; Murai, A.; Horio, F. Genetic analysis of abdominal fat distribution in SM/J and A/J mice. J. Lipid Res. 2010, 51, 3463-3469. [CrossRef] [PubMed]

4. Sarjeant, K.; Stephens, K. Cold Spring Harbor Perspect. Biol 2012, 4, 1485-1495.

5. Ferré, P. The biology of peroxisome proliferator-activated receptors: Relationship with lipid metabolism and insulin sensitivity. Diabetes 2004, 53, S43-S50. [CrossRef] [PubMed]

6. Hooper, L.V.; Wong, M.H.; Thelin, A.; Hansson, L.; Falk, P.G.; Gordon, J.I. Molecular analysis of commensal host-microbial relationships in the intestine. Science 2001, 291, 881-884. [CrossRef] [PubMed]

7. Almeida-Suhett, C.P.; Scott, J.M.; Graham, A.; Chen, Y.; Deuster, P.A. Control diet in a high-fat diet study in mice: Regular chow and purified low-fat diet have similar effects on phenotypic, metabolic, and behavioral outcomes. Nutr. Neurosci. 2019, 22, 19-28. [CrossRef]

8. Nguyen, T.D.; Prykhodko, O.; Hållenius, F.F.; Nyman, M. Monobutyrin reduces liver cholesterol and improves intestinal barrier function in rats fed high-fat diets. Nutrients 2019, 11, 308. [CrossRef]

9. Iván, J.; Major, E.; Sipos, A.; Kovács, K.; Horváth, D.; Tamás, I.; Bay, P.; Dombrádi, V.; Lontay, B. The short-chain fatty acid propionate inhibits adipogenic differentiation of human chorion-derived mesenchymal stem cells through the free fatty acid receptor 2. Stem Cells Dev. 2017, 26, 1724-1733. [CrossRef]

10. Ulven, T. Short-chain free fatty acid receptors FFA2/GPR43 and FFA3/GPR41 as new potential therapeutic targets. Front. Endocrinol. 2012, 3, 111. [CrossRef]

11. Jocken, J.W.; González Hernández, M.A.; Hoebers, N.T.; van der Beek, C.M.; Essers, Y.P.; Blaak, E.E.; Canfora, E.E. Short-chain fatty acids differentially affect intracellular lipolysis in a human white adipocyte model. Front. Endocrinol. 2018, 8, 372. [CrossRef] [PubMed]

12. Hague, A.; Butt, A.J.; Paraskeva, C. The role of butyrate in human colonic epithelial cells: An energy source or inducer of differentiation and apoptosis? Proc. Nutr. Soc. 1996, 55, 937-943. [CrossRef] [PubMed]

13. Chen, S.; Li, X.; Liu, L.; Liu, C.; Han, X. Ophiopogonin D alleviates high-fat diet-induced metabolic syndrome and changes the structure of gut microbiota in mice. FASEB J. 2018, 32, 1139-1153. [CrossRef] [PubMed] 
14. Soetaert, W.; Schwengers, D.; Buchholz, K.; Vandamme, E. A wide range of carbohydrate modifications by a single micro-organism: Leuconostoc mesenteroides. In Progress in Biotechnology; Elsevier: Amsterdam, The Netherlands, 1995; Volume 10, pp. 351-358.

15. Hemme, D.; Foucaud-Scheunemann, C. Leuconostoc, characteristics, use in dairy technology and prospects in functional foods. Int. Dairy J. 2004, 14, 467-494. [CrossRef]

16. Traisaeng, S.; Batsukh, A.; Chuang, T.-H.; Herr, D.R.; Huang, Y.-F.; Chimeddorj, B.; Huang, C.-M. Leuconostoc mesenteroides fermentation produces butyric acid and mediates Ffar2 to regulate blood glucose and insulin in type 1 diabetic mice. Sci. Rep. 2020, 10,1-10. [CrossRef]

17. Kraus, N.A.; Ehebauer, F.; Zapp, B.; Rudolphi, B.; Kraus, B.J.; Kraus, D. Quantitative assessment of adipocyte differentiation in cell culture. Adipocyte 2016, 5, 351-358. [CrossRef]

18. Akiba, Y.; Maruta, K.; Narimatsu, K.; Said, H.; Kaji, I.; Kuri, A.; Iwamoto, K.-i.; Kuwahara, A.; Kaunitz, J.D. FFA2 activation combined with ulcerogenic COX inhibition induces duodenal mucosal injury via the 5-HT pathway in rats. Am. J. Physiol. Gastrl. 2017, 313, G117-G128. [CrossRef]

19. Sikder, K.; Shukla, S.K.; Patel, N.; Singh, H.; Rafiq, K. High fat diet upregulates fatty acid oxidation and ketogenesis via intervention of PPAR- $\gamma$. Cell. Physiol. Biochem. 2018, 48, 1317-1331. [CrossRef]

20. Onyszkiewicz, M.; Gawrys-Kopczynska, M.; Konopelski, P.; Aleksandrowicz, M.; Sawicka, A.; Koźniewska, E.; Samborowska, E.; Ufnal, M. Butyric acid, a gut bacteria metabolite, lowers arterial blood pressure via colon-vagus nerve signaling and GPR41/43 receptors. Pflügers Arch. Eur. J. Physiol. 2019, 471, 1441-1453. [CrossRef]

21. Johanningsmeier, S.; McFeeters, R.F.; Fleming, H.P.; Thompson, R.L. Effects of Leuconostoc mesenteroides starter culture on fermentation of cabbage with reduced salt concentrations. J. Food Sci. 2007, 72, M166-M172. [CrossRef]

22. Liu, S.; Ren, F.; Zhao, L.; Jiang, L.; Hao, Y.; Jin, J.; Zhang, M.; Guo, H.; Lei, X.; Sun, E. Starch and starch hydrolysates are favorable carbon sources for Bifidobacteria in the human gut. BMC Microbiol. 2015, 15, 54. [CrossRef] [PubMed]

23. Hall, J.E. Guyton and Hall Textbook of Medical Physiology e-Book; Elsevier Health Sciences: Amsterdam, The Netherlands, 2010.

24. Nelson, D.L.; Cox, M.M.; Lehninger, A.L. Principles of Biochemistry; Freeman New York: New York, NY, USA, 2008.

25. Kimura, I.; Ozawa, K.; Inoue, D.; Imamura, T.; Kimura, K.; Maeda, T.; Terasawa, K.; Kashihara, D.; Hirano, K.; Tani, T. The gut microbiota suppresses insulin-mediated fat accumulation via the short-chain fatty acid receptor GPR43. Nat. Commun. 2013, 4, 1-12. [CrossRef] [PubMed]

26. Lu, Y.; Fan, C.; Li, P.; Lu, Y.; Chang, X.; Qi, K. Short chain fatty acids prevent high-fat-diet-induced obesity in mice by regulating $G$ protein-coupled receptors and gut microbiota. Sci. Rep. 2016, 6, 1-13. [CrossRef] [PubMed]

27. Cani, P.D.; Amar, J.; Iglesias, M.A.; Poggi, M.; Knauf, C.; Bastelica, D.; Neyrinck, A.M.; Fava, F.; Tuohy, K.M.; Chabo, C. Metabolic endotoxemia initiates obesity and insulin resistance. Diabetes 2007, 56, 1761-1772. [CrossRef]

28. Hickey, M.; Carey, J.; Azevedo, J.; Houmard, J.; Pories, W.; Israel, R.; Dohm, G. Skeletal muscle fiber composition is related to adiposity and in vitro glucose transport rate in humans. Am. J. Physiol. Endocrinol. Metab. 1995, 268, E453-E457. [CrossRef]

29. McNabney, S.M.; Henagan, T.M. Short chain fatty acids in the colon and peripheral tissues: A focus on butyrate, colon cancer, obesity and insulin resistance. Nutrients 2017, 9, 1348. [CrossRef]

30. Pace, B.S.; White, G.L.; Dover, G.J.; Boosalis, M.S.; Faller, D.V.; Perrine, S.P. Short-chain fatty acid derivatives induce fetal globin expression and erythropoiesis in vivo. Blood J. Am. Soc. Hematol. 2002, 100, 4640-4648. [CrossRef]

31. Matheus, V.; Monteiro, L.; Oliveira, R.; Maschio, D.; Collares-Buzato, C. Butyrate reduces high-fat diet-induced metabolic alterations, hepatic steatosis and pancreatic beta cell and intestinal barrier dysfunctions in prediabetic mice. Exp. Biol. Med. 2017, 242, 1214-1226. [CrossRef]

32. Yadav, H.; Lee, J.-H.; Lloyd, J.; Walter, P.; Rane, S.G. Beneficial metabolic effects of a probiotic via butyrate-induced GLP-1 hormone secretion. J. Biol. Chem. 2013, 288, 25088-25097. [CrossRef] 
33. Khan, S.; Jena, G. Protective role of sodium butyrate, a HDAC inhibitor on beta-cell proliferation, function and glucose homeostasis through modulation of p38/ERK MAPK and apoptotic pathways: Study in juvenile diabetic rat. Chem. Biol. Interact. 2014, 213, 1-12. [CrossRef]

34. Heimann, E.; Nyman, M.; Degerman, E. Propionic acid and butyric acid inhibit lipolysis and de novo lipogenesis and increase insulin-stimulated glucose uptake in primary rat adipocytes. Adipocyte 2015, 4, 81-88. [CrossRef] [PubMed]

35. Kubota, N.; Terauchi, Y.; Miki, H.; Tamemoto, H.; Yamauchi, T.; Komeda, K.; Satoh, S.; Nakano, R.; Ishii, C.; Sugiyama, T. PPAR $\gamma$ mediates high-fat diet-induced adipocyte hypertrophy and insulin resistance. Mol. Cell 1999, 4, 597-609. [CrossRef]

36. Dewulf, E.M.; Cani, P.D.; Neyrinck, A.M.; Possemiers, S.; Van Holle, A.; Muccioli, G.G.; Deldicque, L.; Bindels, L.B.; Pachikian, B.D.; Sohet, F.M. Inulin-type fructans with prebiotic properties counteract GPR43 overexpression and PPAR $\gamma$-related adipogenesis in the white adipose tissue of high-fat diet-fed mice. J. Nutr. Biochem. 2011, 22, 712-722. [CrossRef] [PubMed]

37. Poirier, H.; Rouault, C.; Clement, L.; Niot, I.; Monnot, M.-C.; Guerre-Millo, M.; Besnard, P. Hyperinsulinaemia triggered by dietary conjugated linoleic acid is associated with a decrease in leptin and adiponectin plasma levels and pancreatic beta cell hyperplasia in the mouse. Diabetologia 2005, 48, 1059-1065. [CrossRef] [PubMed]

38. Ibrahim, M.M. Subcutaneous and visceral adipose tissue: Structural and functional differences. Obes. Rev. 2010, 11, 11-18. [CrossRef] [PubMed]

39. Kadowaki, T.; Hara, K.; Yamauchi, T.; Terauchi, Y.; Tobe, K.; Nagai, R. Molecular mechanism of insulin resistance and obesity. Exp. Biol. Med. 2003, 228, 1111-1117. [CrossRef]

40. Roberts, L.D.; Murray, A.J.; Menassa, D.; Ashmore, T.; Nicholls, A.W.; Griffin, J.L. The contrasting roles of PPAR $\delta$ and PPAR $\gamma$ in regulating the metabolic switch between oxidation and storage of fats in white adipose tissue. Genome Biol. 2011, 12, R75. [CrossRef]

41. Hong, Y.-H.; Nishimura, Y.; Hishikawa, D.; Tsuzuki, H.; Miyahara, H.; Gotoh, C.; Choi, K.-C.; Feng, D.D.; Chen, C.; Lee, H.-G. Acetate and propionate short chain fatty acids stimulate adipogenesis via GPCR43. Endocrinology 2005, 146, 5092-5099. [CrossRef]

42. Ge,H.; Li, X.; Weiszmann, J.; Wang, P.; Baribault, H.; Chen, J.-L.; Tian, H.; Li, Y. Activation of G protein-coupled receptor 43 in adipocytes leads to inhibition of lipolysis and suppression of plasma free fatty acids. Endocrinology 2008, 149, 4519-4526. [CrossRef]

43. Tilg, H.; Kaser, A. Gut microbiome, obesity, and metabolic dysfunction. J. Clin. Investig. 2011, 121, $2126-2132$. [CrossRef]

44. Everard, A.; Matamoros, S.; Geurts, L.; Delzenne, N.M.; Cani, P.D. Saccharomyces boulardii administration changes gut microbiota and reduces hepatic steatosis, low-grade inflammation, and fat mass in obese and type 2 diabetic $\mathrm{db} / \mathrm{db}$ mice. MBio 2014, 5, e01011-e01014. [CrossRef] [PubMed] 